\title{
Dengue Virus Cellular Receptors and Tropism
}

\author{
Jorge Reyes-del Valle • Juan Salas-Benito • \\ Rubén Soto-Acosta $\cdot$ Rosa M. del Angel
}

Published online: 3 January 2014

(C) Springer International Publishing AG 2014

\begin{abstract}
Viral entry into host cells primordially defines tropism and represents an attractive target to counteract infection either by antiviral agents or by immune mediated mechanisms. Research on Dengue virus entry presents interesting challenges. Whatever the mechanism dengue virus exploits to gain entry into cells, this had to be evolutionarily conserved, so that it is now present in arthropod and human cells. Until now, dengue cellular receptors were not completely unraveled. However, we have clues about the key steps dengue virus is relying on. Initially a group of factors that interact with the virus through carbohydrate interaction assure its adherence and further contact with a protein receptor complex, which is held together thanks to its special interaction with cell membrane lipidic platforms. This interaction may be so intimate that it may trigger not only viral entry through receptormediated endocytosis, but also activation of cell signaling pathways that the virus is going to subvert to its advantage.
\end{abstract}

J. Reyes-del Valle

School of Life Sciences, Arizona State University, LSE-650 427 East

Tyler Mall, Tempe, AZ 85287, USA

e-mail: Jorge.Reyesdelvalle@asu.edu

\section{J. Salas-Benito}

Programa Institucional de Biomedicina Molecular, Escuela Nacional de Medicina y Homeopatía, Instituto Politécnico Nacional,

Guillermo Massieu Helguera 239, Fraccionamiento La

Escalera-Ticomán, Mexico, DF CP 07320, Mexico

e-mail: jsalasb@yahoo.com

R. Soto-Acosta $\cdot$ R. M. del Angel $(\bowtie)$

Departamento de Infectómica y Patogénesis Molecular,

CINVESTAV-IPN, Av. IPN 2508. Col. San Pedro Zacatenco,

México, DF CP 07360, Mexico

e-mail: rubensoto12@hotmail.com

R. M. del Angel

e-mail: rmangel@cinvestav.mx
Keywords Dengue $\cdot$ Receptor $\cdot$ Tropism $\cdot$ Viral entry $\cdot$ Viral tropical medicine $\cdot$ Virus $\cdot$ Dengue cellular receptor

\section{Introduction}

Importance of Dengue Virus Infection

The increase in dengue's worldwide prevalence has made this disease the most important mosquito-borne viral infection for the past six decades. Dengue virus (DENV) inflicts a significant health burden in endemic countries, among the most populated areas worldwide. According to the World Health Organization (WHO), Southeast Asia and southwest Pacific regions reported nearly $75 \%$ of the total cases of dengue infections (WHO, 2009). With an annual average of 574,000 cases reported, the economic cost to eight countries in America and Asia is at least $\$ 587$ million [1]. Indeed, WHO classifies dengue as a major international public health problem [2].

DENV has one of the simplest infectious cycles among arthropod-borne viruses. A person gets the virus from an infected mosquito of the Aedes genus. After a replication period of seven to ten days, DENV reaches levels in peripheral blood (viremia) that allow transmission to an uninfected mosquito. After infecting its digestive tract and circulatory system, DENV reaches the vector salivary glands and the cycle repeats. Dengue prevalence has increased not only because geographic areas where Aedes mosquito dwells coincide with unplanned human settlements, but also because of mutations in circulating DENV and climatic factors [3]. It follows then, that the analysis of DENV entry into host cells of both, human and mosquito nature is of paramount importance for the design of control strategies. 


\section{Dengue Virus Tropism}

Human cells In vitro DENV is able to infect a large variety of cell lines from murine, hamster, canine, primate, and naturally mosquito and human hosts lineages. However, in vivo, few human cell types have been shown to support DENV replication. Samples from patients traditionally come from complicated, severe cases and not from initial phases of infection. In consequence, histopathological studies from patients' biopsies documenting the localization of DENV proteins might be confused with cells that can actively replicate the virus with those cells that acquire dengue antigens by endocytic or phagocytic uptake of viral particles or proteins $[4,5]$. The initial steps following an infected mosquito inoculation have been studied ex vivo, where a skin explant is infected transcutaneusly [6]. DENV is able to infect resident cutaneous Langerhans dendritic cells (DCs) at the site of mosquito inoculation. The infection of these cells results in two important events. First, their activation and migration through the lymphatic system brings DENV to sites where a robust viral replication occurs (secondary lymphoid tissue) and, secondly, in the expression of cytokines and chemokine-mediated recruitment of immune competent cells [6]. The viral infection primary targets in humans and murine models are monocytes/ macrophages, as well as B and T lymphocytes [7-9, 10••]. Although hematopoietic lineage cells are major sites of DENV replication, some other cells of non-hematopoietic origin such as hepatocytes and Kupffer cells or neurons and microglia can be permissive to DENV during natural infection $[5,11]$. Furthermore, autopsies have documented infection of endothelial cells (ECs), a finding that has been reproduced in a mouse model of DENV infection [4, 12]. Infection of ECs can contribute to the pathogenesis of the disease by increasing viremia, cytokine secretion, and modulating host's immune responses [13•]. In this respect, it is not clear if the tropism observed in fatal cases of DENV infection or in the mice model is the same that the one detected in patients with dengue fever $[4,14,15]$.

Mosquito cells Two arthropod vectors from the Aedes genus are responsible of DENV transmission to humans, Ae. aegytpi and Ae. Albopictus [16]. The haematophagous female necessitates a blood meal for oviposition, therefore, Aedes mosquitos usually breed in close proximity to humans. This fact has enormous implications for DENV epidemiology and prevention strategies. The first mosquito organ where DENV replicates is the midgut. Then the virus spreads using the circulatory system to other organs/tissues such as the tracheal system, fat body, salivary glands, nervous system, esophagus, hemocytes, ommatidia of compound eyes, and malphigian tubules [17]. This period of replication that is to reach the salivary glands is known as intrinsic incubation period and lasts approximately five to seven days, afterwards the mosquito becomes a life-long potential virus transmitter.

\section{Dengue Virus Receptors in Human Cells}

Dengue virus entry into human cells The first step in any viral life cycle is viral entry. This dynamic process is usually carried on in multiple steps and the nature of each step depends on the particular characteristic of the virus (depicted in Fig. 1). DENV is an enveloped virus, with a major glycoprotein (E), arranged in ninety dimers with a quasi-icosahedral symmetry. The structure of DENV is very conserved in the Flaviridae family, which has prompted several groups to postulate that the entry process is conserved, but not the particular players. The viral membrane derives from the previous infected cell plasmatic membrane, and it is possible that the lipid components budded off with the virus to play a role to facilitate the process of viral entry [18•], which in the case of dengue and other flaviviruses, is rather fast and estimated to occur in minutes [19]. The study of viral receptors traditionally starts with a susceptible cell line and through biochemical or genetic methods a surface molecule(s) is identified and postulated as putative receptors. Next, if the experimental induction of expression of the putative molecule in non-permissive cells renders them susceptible to infection, the putative molecule is considered a bona fide cell receptor. This process has found some hurdles for DENV and other arthropod borne flaviviruses. The range of susceptibility of many cell lines of different origins is very wide, and finding a non-susceptible cell line has proven to be a non-trivial task. On the other hand, the molecules identified to be putative receptors are either of not a protein nature (hence, no induction of expression) or with an important intracellular housekeeping function besides their potential role on the cell membrane (for instance surface chaperones). In sum, no single protein or proteins have been singled out as the DENV cell receptor, and what follows is a recounting of the molecules that have been implicated in DENV entry into mammalian cells.

Glycosaminoglycans (GAGs) and other attachment factors The first molecule that was identified to participate in DENV entry into mammalian cells was heparan sulfate [20-22]. Highly sulfated GAGs are ubiquitous molecules present on the surface of several types of cells, also mediating attachment for many viruses [23]. It is documented that there are electrostatic attractions among a dengue virus E glycoprotein and the negatively charged carbohydrate moieties present in GAGs [24]. Due to the documented evidence that the GAGs-DENV interactions are stronger when the virus has been passaged in cell-culture repetitively, and due to its correlation with in vivo attenuation, it has been suggested its role is artifactual. The most relevant attachment factor for DENV entry identified so far is the calcium-dependent lectin, 


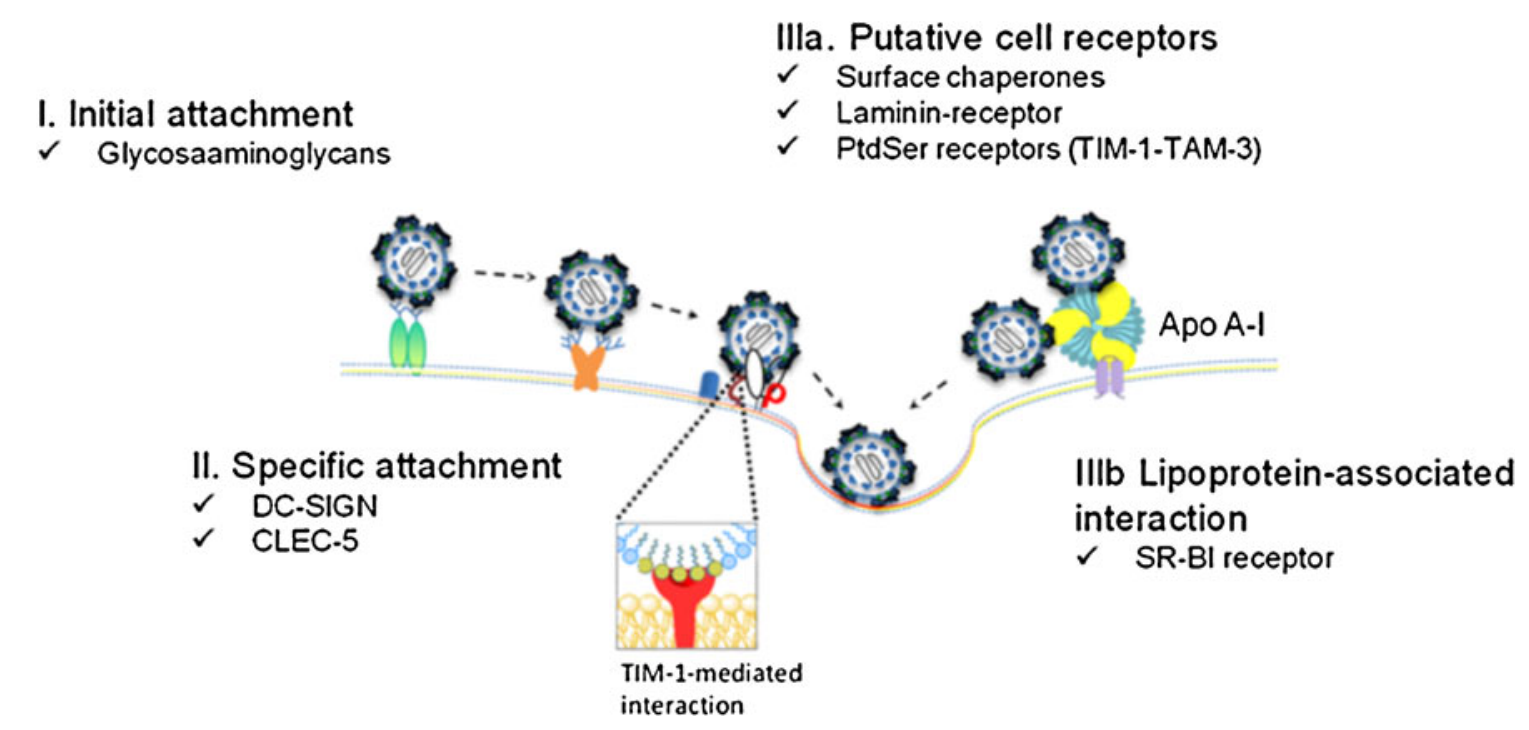

Fig. 1 The process in multiple steps and the nature of each step depends on the particular characteristic of the virus

dendritic cell-specific intercellular adhesion molecule 3grabbing non-integrin (DC-SIGN) [25-27]. This receptor has high affinity for high-mannose ligands [28]. During the mosquito blood-meal, DENV is deposited in the dermis where its interaction with resident dendritic cells (Langerhans cells) has been documented [6]. Upon entry, dendritic cells migrate to draining lymph nodes where the infection is spread to immune-competent myelo-monocytic cells. Whether DCSIGN is the only factor responsible for dengue virus entry or the virus replicates actively in dendritic cells is still unknown. Another lectin that may play a role in dengue virus attachment is the C-type lectin domain family-5 member A (CLEC5) [29, 30]. This molecule has been documented to participate in viral attachment and release of pro-inflammatory mediators important in the pathogenesis of severe forms of dengue [31, 32]. In sum, it is clear that the first step in dengue virus entry involves attachment of the virion to the cell surface.

Cell surface chaperones Among DENV entry factors identified thus far, there is a group that has been described by independent groups, the surface chaperones. Experimentally, these molecules have been found through binding assays using the envelope glycoprotein as a ligand. HSP-90, HSP70 and GRP-78 are thought to be part of a receptor complex that mediates dengue entry into human cells from monocytic, neural and hepatic origin [33, 34]. It is not known whether their extracellular function is similar to their well-known intracellular, ATP-dependent protein folding activity, or how they associate with the cell membrane. It has been long been reported that lipopolysaccharide (LPS) inhibits DENV entry into monocytes [35]. This effect is dose dependent. On this regard, surface HSPs were also documented as part of an interacting cluster of plasmatic proteins mediating physiological effects of LPS sensing on cells [36]. It is tempting to propose that heat shock as an effect on DENV entry through the increase of heat-shock-responsive molecules on the surface of infected cells. This effect was documented in the infection of a monocytic cell line [37]. Another protein with chaperone function reported as a DENV receptor is the protein disulfide isomerase (PDI). This has been implicated in DENV entry into endothelial cells [38•]. Besides its important role in the endoplasmic reticulum, it has been detected on the cell surface of lymphocytes [39], platelets [40, 41], and endothelial cells [38 $]$. PDI has been implicated in entry of several viruses such as polyomavirus [42], New Castle disease virus [43], and the human immunodeficiency virus [39, 44, 45].

Lipid receptors The only gain-of-function screening in the search for DENV receptors found that phosphatydilserine (PtdSer) receptors TIM-1 (T cell immunoglobulin domain and mucin domain) directly and indirectly through TAM ligands, are relevant on DENV entry into the human $293 \mathrm{~T}$ cell line [46•]. These receptors mediate binding of apoptotic cells to be scavenged and immunotolerance. Therefore, an intimate interaction of cell receptors with lipids present on the virion membrane envelope suggests the structure of entering particles needs to be revisited. In any case, the lipid content present on the target cell has long been demonstrated as important to optimize DENV entry.

\section{Lipids in DENV Entry}

Role of lipid rafts in dengue virus entry Recent evidence suggests that lipid rafts, defined as detergent resistant membrane microdomains enriched in cholesterol and sphingolipids [47], might serve as platforms to concentrate virus receptors. Since several of the DENV cell receptors are residents of lipid rafts or are relocated to them after DENV binding [48-50], 
these platforms traffic the virus to a proper intracellular environment to favor conformational changes in the envelope proteins during the fusion process $[51,52]$.

The role of lipid rafts in DENV entry has been studied in several mammalian cell lines. In macrophages it has been demonstrated that lipid rafts play an important role in DENV entry in the absence and also in the presence of facilitating antibodies [33, 53••]. The cholesterol dependence of DENV entry and post entry steps was also observed in the mouse neuroblastoma cell line N18, in the hepatocarcinoma cell line Huh $7[54,55]$ and in the endothelial cell line HMEC-1 [56].

However, the involvement of cholesterol in DENV entry does not seem to be a general event, because it has been described that, for green monkey epithelial kidney cell lines (Vero and BS-C-1 cells), hepatocarcinoma cell line HepG2 and ECV304 cells, cholesterol is not required [18•, 19, 57-59]. Furthermore, contradictory data exist about the cholesterol dependence in U937, K562 (hematopoietic) and A549 cells (lung carcinoma) $[18 \cdot, 33,57,60]$. On the other hand, DENV entry in the mosquito cell line C6/36 seems to be cholesterol and lipid rafts independent because pretreatment of these cells with lipid rafts disrupters $(\mathrm{M} \beta \mathrm{CD}$, filipin and Nystatin) did not inhibit DENV 1 and 2 infection [61, 62]. Similarly, it has been observed that the infection and fusion capacities of DENV remain unaltered in a cholesterol depleted-insect cell system [63].

Role of lipids in viral fusion Several observations have suggested that, in addition to low $\mathrm{pH}$, lipid cofactors are required for DENV fusion. The outer leaflets of the plasma membranes of mosquito cells and the membranes of late endosomes of mammalian cells have high concentrations of the anionic lipids phophatidylserine (PS) and bis(monoacylglycero)phosphate (BMP), respectively. It has been demonstrated that the DENV fusion occurs successfully only upon the addition of anionic lipids (PS and BMP) to liposomal, plasma and intracellular membranes, suggesting that when DENV get into contact with the late endosomes membranes containing anionic lipids, the envelope protein (E) acquires its fusogenic conformation by action of endosomes acidification $[64 \bullet \bullet]$.

On the other hand, the addition of cholesterol to artificial receptor-free lipid membranes (liposomes) consisting of phosphatidylcholine and phosphatidylethanolamine, increases the membrane fusion capacity of DENV, Tick Borne Encephalitis Virus (TBEV) and West Nile Virus (WNV) at low pH [65••]. Furthermore, the role of cholesterol in viral trafficking was evidenced using U18666A, a cholesterol transport inhibitor, which causes a delay in the release of viral genome into the cytoplasm [66•].
Finally, lipids present on the DENV envelope, specifically cholesterol and PS, have also been involved in the fusion process. Carro et al., demonstrated that the cholesterol present in the viral particle is a critical factor during fusion process because DENV binding and entry is inhibited when viral particles were treated with $\mathrm{M} \beta C D$ [18•]. Similarly, as mentioned by Meertens et al., PS present on DENV particles surface is recognized directly by TIM proteins and indirectly by TAM proteins that mediate the PS-dependent phagocytic removal of apoptotic cells and can be exploited by the virus as entry factors $\left[46^{\bullet}\right]$.

Role of lipoproteins in viral infection Recently, it has been reported that DENV C protein is able to interact with Very Low Density Lipoprotein (VLDL), specifically with Apo E, which is the main surface apolipoprotein in VLDL. This fact is supported by the strong structural similarities between Apo E and perilipin 3, the DENV C protein ligand in lipid droplets $[67 \cdot, 68]$. It has been proposed that the interaction between $\mathrm{C}$ and VLDL ApoE may allow the formation of Lipoviroparticles (LVP), as has been reported for HCV [69]. They might be transported to the extracellular medium and infect neighboring cells through lipoproteins receptors. However, the presence of LVP during DENV infection in vivo has not been observed, and the direct role of VLDL or LDL receptors in DENV entry has not been determined. On the contrary, there is a report where the interaction of the apolipoprotein A-I (Apo A-1), the main protein component in High Density Lipoprotein (HDL) with DENV particle was confirmed by co-immunoprecipitation assays [70 0 . The authors suggest that this interaction is able to promote DENV infection through Apo A-I receptor, the Scavenger Receptor class B type I (SR-BI), and provides insights into the functional importance of lipoproteins in dengue pathogenesis.

\section{Dengue Virus Receptors in Mosquitoes}

The fact that DENV enters mosquito cells as efficiently as mammalian cells implies that the molecules, strategies, or mechanisms used by the virus are either very conserved or fungible. Nevertheless, DENV is very selective regarding the arthropod it uses, only mosquitoes from the Aedes genus are susceptible. This is by no means a rule for other arthropodborne viruses from the Flaviviridae family, like the West Nile virus (WNV), or the Japanese encephalitis virus (JEV), which are more "promiscuous" in this regard. Next, a brief description of some of the molecules identified as putative DENV receptors in mosquito cells is offered (see Table 1).

Heparan sulphate Treatment of $\mathrm{C} 6 / 36$ cells (a line derived from Ae. Albopictus larva, [71] with heparinase III prior to infection with the four DENV serotypes showed a variable inhibition, from $25 \%$ for DENV 2 to $60 \%$ for DENV 1 [72]. 
Table 1 Summary of the putative receptors for DENV identified in mosquitoes or mosquito cells

\begin{tabular}{llll}
\hline Receptor & $\begin{array}{l}\text { DENV } \\
\text { Serotype }\end{array}$ & Model & Reference \\
\hline $\begin{array}{l}\text { Hepara sulfate } \\
\text { high-affinity laminin related } \\
\text { receptor }\end{array}$ & $\begin{array}{l}\text { 1,2,3, and } 4 \\
\text { Prohibitin }\end{array}$ & C6/36 cells & {$[72]$} \\
HSP70 & 2 & C6/36 cells & {$[72]$} \\
Hsc70 & 4 & CCL-125 and C6/36 cells Ae aegypti & {$[76 \bullet]$} \\
GRP78 (BiP) & 2 & C6/36 cells & {$[83 \bullet]$} \\
PDI & 4 & A7 and C6/36 cells Ae aegypti mosquitoes & {$[75]$} \\
Vav-1 & 4 & C6/36 cells & {$[83 \bullet]$} \\
ATP synthase $\beta$ subunit & 2 & C6/36 cells & {$[83 \bullet]$} \\
Orisis & 2 & A7 and C6/36 cells Ae aegypti mosquitoes & {$[75]$} \\
67 and $80 \mathrm{kDa}$ proteins & 2 & A7 and C6/36 cells Ae aegypti mosquitoes & {$[75]$} \\
& $1,2,3$, and & C6/36 cells Ae. Aegypti mosquitoes & {$[75]$} \\
& 4 & & {$[94]$} \\
$77,58,54$, and $37 \mathrm{kDa}$ proteins & $1,2,3$ and 4 & Ae. Aegypti mosquitoes & {$[93]$} \\
$67,56,54,50$, and $48 \mathrm{kDa}$ & $1,2,3$ and 4 & Ae. Polynesiensis mosquitoes & {$[95]$} \\
proteins & & & {$[96]$} \\
\hline
\end{tabular}

Interestingly, a similar treatment reduced $40 \%$ the infectivity of JEV [73] whereas treatment with glycosidases, heparinases, lectins and phospholipases did not affect the infection by WNV [74] suggesting they use different molecules for cell surface attachment in mosquito cells.

Laminin receptor Homology with the $37 / 67-\mathrm{kDa}$ human high-affinity laminin-like receptor was documented in a 50$\mathrm{kDa}$ protein present in C6/36 cells that binds DENV 2, 3 and 4. To support its role as receptor, immune sera raised against this molecule or soluble laminin was able to inhibit the infectivity of the four serotypes of DENV with differing efficiency [72]. In contrast, soluble laminin only reduced JEV infection up to $20-30 \%$ in $\mathrm{C} 6 / 36$ cells suggesting that this receptor plays a minor role for JEV internalization [73].

Prohibitin Recently, two independent research groups identified the protein prohibitin (PHB) as a receptor for DENV 2 in cells lines from A. albopitcus and A. aegyptii (C6/36, CCL-125, and A7, respectively) as well as in midgut mosquito cells $[75,76 \cdot$. Silencing of prohibitin with specific siRNA reduced virus infection in both mosquito cell lines, and specific immune sera competed with both virus infection and cell binding. PHB is a ubiquitously expressed and highly conserved protein complex in eukaryotic cells composed of two proteins: PHB1 $(\sim 30 \mathrm{kDa})$ and PHB2 $(\sim 37 \mathrm{kDa})$ [77]. They have more than $50 \%$ amino-acid identity and are prone to form oligomers that have been proposed to enhance the stability of the complex [77, 78]. PHB is present in many compartments of the cell, mainly the mitochondria [79] but it is also present in the cytoplasm and nucleus [78]. Early evidence indicated that this protein acts as an inhibitor of cell proliferation, and that it plays a role in cell signaling at the plasma membrane and in transcriptional regulation [80]. Interestingly, it has also been described as a cell receptor for the Chikungunya virus (CHIKV), an arthropod borne alphavirus, in microglial cells $[81 \bullet]$.

Chaperones and heat shock-related proteins Similarly to mammalian cells, C6/36 cells increase their susceptibility to DENV upon heat shock (HS). A 74-kDa protein that binds DENV 2 and is strongly expressed under HS conditions [82] was recently isolated and identified as three proteins: $78-\mathrm{kDa}$ glucose-regulated protein (GRP78 or BiP), the $70-\mathrm{kDa}$ heat shock protein (HSP70) and the $70-\mathrm{kD}$ heat shock cognate protein (HSC70) [83•]. Specific antibodies against GRP78, and HSP70/HSC70 were able to inhibit DENV4 infectivity. These proteins are ER resident chaperones of the HSP70 family $[84,85]$. They are located ubiquitously in cytoplasm, mitochondria, nucleus and plasma membrane [86-88]. Independently, another group also found HSC70 interacting with DENV2 in C6/36 and A7 cells as well as in Ae aegypti females [75]. Furthermore, the chaperone HSC70 has been implicated in JEV entry into C6/36 [89] and mammalian cells [90]. Additionally, a protein $(40-\mathrm{kDa})$ with homology to protein disulfide isomerase (PDI) was also identified among the C6/ 36 molecules that interacted with DENV4 by affinity chromatography [83•]. Interestingly, PDI has been implicated in DENV entry to endothelial cells [38 $]$ and is also a putative 
receptor for other viruses (see above). Several groups through different approaches have documented the participation of cell-surface chaperone proteins in dengue virus entry into mosquito cells.

Unidentified proteins Several other proteins in mosquito cell lines or midgut cells from competent vectors have been proposed to function as receptors for DENV. Among them, a $45-\mathrm{kDa}$ glycoprotein present on the surface of C6/36 was recognized by DENV4 by virus overlay protein blot assay (VOPBA) [91]. Its presence was also documented in extracts of different development stages of Ae. aegypti mosquitoes as well as in different susceptible mosquito tissues (salivary gland, ovaries and midgut), but not in a non-dengue vector as Anopheles albimanus, suggesting the participation of this molecule in DENV infection as well as in vertical transmission [92]. Other groups reported molecules with weights of 67 and $80 \mathrm{kDa}$ important for DENV2 entry [93, 94]. Interestingly, the presence of the $67 \mathrm{kDa}$ protein in midgut cells of three varieties of Ae. aegypti mosquitoes correlated with their susceptibility to DENV infection, thus, it has been proposed as a vector competence marker [95]. Finally, proteins ranging in molecular weight from 77 to $37 \mathrm{kDa}$ obtained from Ae. aegypti and Ae. polynesiensis salivary glands extracts were identified to interact with the four DENV serotypes. Interestingly, proteins with similar molecular weights were recognized by DENV 4 clinical isolate, suggesting that no modification in receptor usage occurred in viruses propagated for a long time in vitro [96].

\section{Conclusions}

Arthropod-borne viruses constitute an excellent model to study evolutionarily conserved mechanisms exploited for a virus to gain cell entry. Even though specific steps in DENV entry have been progressively well characterized in recent years, the specific role of host cell proteins in viral attachment and entry is not completely understood. However, it is possible to infer that a group of factors that interact with the virus through carbohydrate interaction assure its adherence and further contact with a protein receptor complex, which is held together thanks to its special interaction on lipidic platforms on the cell membrane. All these steps allow viral particle entry into early endosomes very rapidly. Chaperones constitute one of the host cell proteins used for DENV in both mammalian and insect cells. The use of several molecules during viral attachment and entry reveal the outstanding requirements and the complex mechanisms that DENV employs to enter the cell. Further studies directed to understand the initial steps in DENV entry have the potential to provide insights into possible therapeutic targets to control DENV infection.

\section{Compliance with Ethics Guidelines}

Conflict of Interest Jorge Reyes-del Valle, Juan Salas-Benito, Rubén Soto-Acosta, and Rosa M. del Angel declare that they have no conflict of interest

Human and Animal Rights and Informed Consent This article does not contain any studies with human or animal subjects performed by any of the authors.

\section{References}

Papers of particular interest, published recently, have been highlighted as:

- Of importance

•- Of major importance

1. Suaya JA et al. Cost of dengue cases in eight countries in the Americas and Asia: a prospective study. Am J Trop Med Hyg. 2009;80(5):846-55.

2. Wilder-Smith $\mathrm{A}$ et al. Update on dengue: epidemiology, virus evolution, antiviral drugs, and vaccine development. Curr Infect Dis Rep. 2010;12(3):157-64.

3. Wilder-Smith A, Gubler DJ. Geographic expansion of dengue: the impact of international travel. Med Clin North Am. 2008;92(6): 1377-90. x.

4. Balsitis SJ et al. Tropism of dengue virus in mice and humans defined by viral nonstructural protein 3-specific immunostaining. Am J Trop Med Hyg. 2009;80(3):416-24.

5. Jessie $\mathrm{K}$ et al. Localization of dengue virus in naturally infected human tissues, by immunohistochemistry and in situ hybridization. J Infect Dis. 2004;189(8):1411-8.

6. Wu SJ et al. Human skin Langerhans cells are targets of dengue virus infection. Nat Med. 2000;6(7):816-20.

7. Blackley $S$ et al. Primary human splenic macrophages, but not $T$ or $\mathrm{B}$ cells, are the principal target cells for dengue virus infection in vitro. J Virol. 2007;81(24):13325-34.

8. Martins Sde Tet al. Dendritic cell apoptosis and the pathogenesis of dengue. Viruses. 2012;4(11):2736-53.

9. King $\mathrm{AD}$ et al. B cells are the principal circulating mononuclear cells infected by dengue virus. Southeast Asian J Trop Med Public Health. 1999;30(4):718-28.

10.• Mota J, Rico-Hesse R. Dengue virus tropism in humanized mice recapitulates human dengue fever. PLoS One. 2011;6(6):e20762. This paper contains the description of a humanized mice that represents one of the two animal models used for dengue virus infection.

11. Huerre MR et al. Liver histopathology and biological correlates in five cases of fatal dengue fever in Vietnamese children. Virchows Arch. 2001;438(2):107-15.

12. Zellweger RM, Prestwood TR, Shresta S. Enhanced infection of liver sinusoidal endothelial cells in a mouse model of antibody-induced severe dengue disease. Cell Host Microbe. 2010;7(2):128-39. 
13. Dalrymple NA, Mackow ER. Endothelial cells elicit immuneenhancing responses to dengue virus infection. J Virol. 2012;86(12):6408-15. In this paper, the authors describe the key role of endothelial cells in dengue virus pathogenesis.

14. Bhoopat L et al. Immunohistochemical characterization of a new monoclonal antibody reactive with dengue virus-infected cells in frozen tissue using immunoperoxidase technique. Asian Pac J Allergy Immunol. 1996;14(2):107-13.

15. Couvelard A et al. Report of a fatal case of dengue infection with hepatitis: demonstration of dengue antigens in hepatocytes and liver apoptosis. Hum Pathol. 1999;30(9):1106-10.

16. Machado CM et al. Epidemiology of neglected tropical diseases in transplant recipients. Review of the literature and experience of a Brazilian HSCT center. Rev Inst Med Trop Sao Paulo. 2009;51(6): 309-24.

17. Salazar MI et al. Dengue virus type 2: replication and tropisms in orally infected Aedes aegypti mosquitoes. BMC Microbiol. 2007;7:9.

18. Carro AC, Damonte EB. Requirement of cholesterol in the viral envelope for dengue virus infection. Virus Res. 2013;174(1-2):7887. The authors present evidence about the importance of the presence of cholesterol in the viral particle.

19. Suksanpaisan L, Susantad T, Smith DR. Characterization of dengue virus entry into HepG2 cells. J Biomed Sci. 2009;16:17.

20. Chen $\mathrm{Y}$ et al. Dengue virus infectivity depends on envelope protein binding to target cell heparan sulfate. Nat Med. 1997;3(8):866-71.

21. Hilgard P, Stockert R. Heparan sulfate proteoglycans initiate dengue virus infection of hepatocytes. Hepatology. 2000;32(5):106977.

22. Dalrymple N, Mackow ER. Productive dengue virus infection of human endothelial cells is directed by heparan sulfate-containing proteoglycan receptors. J Virol. 2011;85(18):9478-85.

23. Akhtar J, Shukla D. Viral entry mechanisms: cellular and viral mediators of herpes simplex virus entry. FEBS J. 2009;276(24): 7228-36.

24. Kovensky J. Sulfated oligosaccharides: new targets for drug development? Curr Med Chem. 2009;16(18):2338-44.

25. Lozach PY et al. Dendritic cell-specific intercellular adhesion molecule 3-grabbing non-integrin (DC-SIGN)-mediated enhancement of dengue virus infection is independent of DC-SIGN internalization signals. J Biol Chem. 2005;280(25):23698-708.

26. Tassaneetrithep B et al. DC-SIGN (CD209) mediates dengue virus infection of human dendritic cells. J Exp Med. 2003;197(7):823-9.

27. Navarro-Sanchez E et al. Dendritic-cell-specific ICAM3-grabbing non-integrin is essential for the productive infection of human dendritic cells by mosquito-cell-derived dengue viruses. EMBO Rep. 2003;4(7):723-8.

28. Modis Y et al. Variable surface epitopes in the crystal structure of dengue virus type 3 envelope glycoprotein. J Virol. 2005;79(2): 1223-31.

29. Chen ST et al. CLEC5A is critical for dengue-virus-induced lethal disease. Nature. 2008;453(7195):672-6.

30. Watson AA, O'Callaghan CA. Crystallization and X-ray diffraction analysis of human CLEC5A (MDL-1), a dengue virus receptor. Acta Crystallogr Sect F: Struct Biol Cryst Commun. 2010;66(Pt 1):29-31.

31. Cheung $\mathrm{R}$ et al. Activation of MDL-1 (CLEC5A) on immature myeloid cells triggers lethal shock in mice. J Clin Invest. 2011;121(11):4446-61.

32. Wu MF, Chen ST, Hsieh SL. Distinct regulation of dengue virusinduced inflammasome activation in human macrophage subsets. J Biomed Sci. 2013;20:36.

33. Reyes-Del Valle $J$ et al. Heat shock protein 90 and heat shock protein 70 are components of dengue virus receptor complex in human cells. J Virol. 2005;79(8):4557-67.

34. Jindadamrongwech $\mathrm{S}$, Thepparit $\mathrm{C}$, Smith DR. Identification of GRP 78 (BiP) as a liver cell expressed receptor element for dengue virus serotype 2. Arch Virol. 2004;149(5):915-27.
35. Chen YC, Wang SY, King CC. Bacterial lipopolysaccharide inhibits dengue virus infection of primary human monocytes/ macrophages by blockade of virus entry via a CD14-dependent mechanism. J Virol. 1999;73(4):2650-7.

36. Triantafilou K, Triantafilou M, Dedrick RL. A CD14-independent LPS receptor cluster. Nat Immunol. 2001;2(4):338-45.

37. Chavez-Salinas $\mathrm{S}$ et al. Heat shock effect upon dengue virus replication into U937 cells. Virus Res. 2008;138(1-2):111-8.

38. Wan SW et al. Endothelial cell surface expression of protein disulfide isomerase activates betal and beta3 integrins and facilitates dengue virus infection. J Cell Biochem. 2012;113(5):1681-91. In this paper, the authors describe the role of protein disulfide isomerase and integrins in the first steps of dengue virus infection into endothelial cells.

39. Barbouche $\mathrm{R}$ et al. Glycosaminoglycans and protein disulfide isomerase-mediated reduction of HIV Env. Mol Pharmacol. 2005;67(4):1111-8

40. Cheng $\mathrm{HJ}$ et al. Anti-dengue virus nonstructural protein 1 antibodies recognize protein disulfide isomerase on platelets and inhibit platelet aggregation. Mol Immunol. 2009;47(2-3):398-406.

41. Holbrook LM et al. Platelets release novel thiol isomerase enzymes which are recruited to the cell surface following activation. Br J Haematol. 2010;148(4):627-37.

42. Gilbert $\mathbf{J}$ et al. Downregulation of protein disulfide isomerase inhibits infection by the mouse polyomavirus. J Virol. 2006;80(21):10868-70.

43. Jain S, McGinnes LW, Morrison TG. Overexpression of thiol/disulfide isomerases enhances membrane fusion directed by the Newcastle disease virus fusion protein. J Virol. 2008;82(24):12039-48.

44. Gallina A et al. Inhibitors of protein-disulfide isomerase prevent cleavage of disulfide bonds in receptor-bound glycoprotein 120 and prevent HIV-1 entry. J Biol Chem. 2002;277(52):50579-88.

45. Wang $Z$ et al. Snapshot of the interaction between HIV envelope glycoprotein 120 and protein disulfide isomerase. Acta Biochim Biophys Sin (Shanghai). 2010;42(5):358-62.

46. Meertens L et al. The TIM and TAM families of phosphatidylserine receptors mediate dengue virus entry. Cell Host Microbe. 2012;12(4):544-57. In this study, authors describe the importance of phosphatidylserine receptors in dengue virus entry into cells.

47. Brown DA, Rose JK. Sorting of GPI-anchored proteins to glycolipid-enriched membrane subdomains during transport to the apical cell surface. Cell. 1992;68(3):533-44.

48. Cambi A et al. Microdomains of the C-type lectin DC-SIGN are portals for virus entry into dendritic cells. J Cell Biol. 2004;164(1): $145-55$.

49. Garcia-Garcia E, Brown EJ, Rosales C. Transmembrane mutations to FcgammaRIIA alter its association with lipid rafts: implications for receptor signaling. J Immunol. 2007;178(5):3048-58.

50. Philippova $\mathrm{M}$ et al. Identification of proteins associating with glycosylphosphatidylinositol- anchored T-cadherin on the surface of vascular endothelial cells: role for $\mathrm{Grp} 78 / \mathrm{BiP}$ in T-cadherindependent cell survival. Mol Cell Biol. 2008;28(12):4004-17.

51. Rawat SS et al. Modulation of entry of enveloped viruses by cholesterol and sphingolipids (Review). Mol Membr Biol. 2003;20(3):243-54.

52. Chazal N, Gerlier D. Virus entry, assembly, budding, and membrane rafts. Microbiol Mol Biol Rev. 2003;67(2):226-37. Table of contents.

53.• Puerta-Guardo $\mathrm{H}$ et al. Antibody-dependent enhancement of dengue virus infection in U937 cells requires cholesterol-rich membrane microdomains. J Gen Virol. 2010;91(Pt 2):394-403. This paper describes for the first time that colesterol rich membrane microdomains are required for dengue virus infection in the presence of facilitating antibodies.

54. Lee CJ et al. Cholesterol effectively blocks entry of flavivirus. J Virol. 2008;82(13):6470-80. 
55. Soto-Acosta $\mathrm{R}$ et al. The increase in cholesterol levels at early stages after dengue virus infection correlates with an augment in LDL particle uptake and HMG-CoA reductase activity. Virology. 2013;442(2):132-47.

56. Martinez-Gutierrez M, Castellanos JE, Gallego-Gomez JC. Statins reduce dengue virus production via decreased virion assembly. Intervirology. 2011;54(4):202-16.

57. Acosta EG, Castilla V, Damonte EB. Alternative infectious entry pathways for dengue virus serotypes into mammalian cells. Cell Microbiol. 2009;11(10):1533-49.

58. van der Schaar HM et al. Dissecting the cell entry pathway of dengue virus by single-particle tracking in living cells. PLoS Pathog. 2008;4(12):e1000244.

59. Peng T et al. Entry of dengue virus serotype 2 into ECV304 cells depends on clathrin-dependent endocytosis, but not on caveolaedependent endocytosis. Can J Microbiol. 2009;55(2):139-45.

60. Rothwell $\mathrm{C}$ et al. Cholesterol biosynthesis modulation regulates dengue viral replication. Virology. 2009;389(1-2):8-19.

61. Acosta EG, Castilla V, Damonte EB. Functional entry of dengue virus into Aedes albopictus mosquito cells is dependent on clathrinmediated endocytosis. J Gen Virol. 2008;89(Pt 2):474-84.

62. Mosso $\mathrm{C}$ et al. Endocytic pathway followed by dengue virus to infect the mosquito cell line C6/36 HT. Virology. 2008;378(1):193-9.

63. Umashankar M et al. Differential cholesterol binding by class II fusion proteins determines membrane fusion properties. J Virol. 2008;82(18):9245-53.

64.• Zaitseva E et al. Dengue virus ensures its fusion in late endosomes using compartment-specific lipids. PLoS Pathog. 2010;6(10): $\mathrm{e} 1001131$. In this paper, authors determine the specific role of some types of lipids in viral entry and specifically in viral fusion.

65.• Smit JM et al. Flavivirus cell entry and membrane fusion. Viruses. 2011;3(2):160-71. This is a very interesting review that describe the first steps in flavivirus entry into the host cells.

66. Poh MK et al. U18666A, an intra-cellular cholesterol transport inhibitor, inhibits dengue virus entry and replication. Antiviral Res. 2012;93(1):191-8. Reveals the relevance of cholesterol by the use of the cholesterol transport inhibitor U18666A.

67. Faustino, A.F., et al., Dengue virus capsid protein interacts specifically with very low-density lipoproteins. Nanomedicine, 2013. In this paper, authors describe the ability of the structural protein $C$ of dengue virus to interact with very low-density lipoproteins.

68. Samsa MM et al. Dengue virus capsid protein usurps lipid droplets for viral particle formation. PLoS Pathog. 2009;5(10):e1000632.

69. Andre $\mathrm{P}$ et al. Characterization of low- and very-low-density hepatitis C virus RNA-containing particles. J Virol. 2002;76(14):6919-28.

70. $\mathrm{Li} \mathrm{Y}$ et al. Human apolipoprotein A-I is associated with dengue virus and enhances virus infection through SR-BI. PLoS One. 2013;8(7):e70390. This paper describe the ability of dengue virus to bind apolipoprotein A-1. This interaction facilitates dengue virus infection.

71. Igarashi A. Isolation of a Singh's Aedes albopictus cell clone sensitive to Dengue and Chikungunya viruses. J Gen Virol. 1978;40(3):531-44.

72. Sakoonwatanyoo P, Boonsanay V, Smith DR. Growth and production of the dengue virus in C6/36 cells and identification of a laminin-binding protein as a candidate serotype 3 and 4 receptor protein. Intervirology. 2006;49(3):161-72.

73. Boonsanay V, Smith DR. Entry into and production of the Japanese encephalitis virus from C6/36 cells. Intervirology. 2007;50(2):85-92.

74. Chu JJ, Leong PW, Ng ML. Characterization of plasma membraneassociated proteins from Aedes albopictus mosquito (C6/36) cells that mediate West Nile virus binding and infection. Virology. 2005;339(2):249-60.

75. Paingankar MS, Gokhale MD, Deobagkar DN. Dengue-2-virusinteracting polypeptides involved in mosquito cell infection. Arch Virol. 2010;155(9):1453-61.
76. Kuadkitkan A et al. Identification and characterization of prohibitin as a receptor protein mediating DENV-2 entry into insect cells. Virology. 2010;406(1):149-61. This paper described the role of the multifunctional cell protein, prohibitin, in dengue virus entry into mosquito cells.

77. Back JW et al. A structure for the yeast prohibitin complex: Structure prediction and evidence from chemical crosslinking and mass spectrometry. Protein Sci. 2002;11(10):2471-8.

78. Mishra S, Murphy LC, Murphy LJ. The Prohibitins: emerging roles in diverse functions. J Cell Mol Med. 2006;10(2):353-63.

79. Artal-Sanz M, Tavernarakis N. Prohibitin and mitochondrial biology. Trends Endocrinol Metab. 2009;20(8):394- 401.

80. Osman C, Merkwirth C, Langer T. Prohibitins and the functional compartmentalization of mitochondrial membranes. J Cell Sci. 2009;122(Pt 21):3823-30.

81. Wintachai $\mathrm{P}$ et al. Identification of prohibitin as a Chikungunya virus receptor protein. J Med Virol. 2012;84(11):1757-70. This paper describes that prohibitin, characterized as a dengue virus receptor, is also functioning as a Chikungunya receptor.

82. Salas-Benito J et al. Evidence that the $45-\mathrm{kD}$ glycoprotein, part of a putative dengue virus receptor complex in the mosquito cell line C6/36, is a heat-shock related protein. Am J Trop Med Hyg. 2007;77(2):283-90

83. Vega-Almeida TO et al. Surface proteins of C6/36 cells involved in dengue virus 4 binding and entry. Arch Virol. 2013;158(6):1189207. This paper describes the identification of a group of proteins, with affinity to E protein, that are involved in dengue virus binding and entry into mosquito cells.

84. Ni M, Lee AS. ER chaperones in mammalian development and human diseases. FEBS Lett. 2007;581(19):3641-51.

85. Schwarze S, Rangnekar VM. Targeting plasma membrane GRP78 for cancer growth inhibition. Cancer Biol Ther. 2010;9(2):153-5.

86. Delpino A et al. Cell surface localization of the $78 \mathrm{kD}$ glucose regulated protein (GRP 78) induced by thapsigargin. Mol Membr Biol. 1998;15(1):21-6.

87. Delpino A, Castelli M. The $78 \mathrm{kDa}$ glucose-regulated protein (GRP78/BIP) is expressed on the cell membrane, is released into cell culture medium and is also present in human peripheral circulation. Biosci Rep. 2002;22(3-4):407-20.

88. Zhang $\mathrm{Y}$ et al. Cell surface relocalization of the endoplasmic reticulum chaperone and unfolded protein response regulator GRP78/ BiP. J Biol Chem. 2010;285(20):15065-75.

89. Ren J et al. Does Japanese encephalitis virus share the same cellular receptor with other mosquito-borne flaviviruses on the C6/36 mosquito cells? Virol J. 2007;4:83.

90. Zhu YZ et al. Association of heat-shock protein 70 with lipid rafts is required for Japanese encephalitis virus infection in Huh7 cells. J Gen Virol. 2012;93(Pt 1):61-71.

91. Salas-Benito JS, del Angel RM. Identification of two surface proteins from $\mathrm{C} 6 / 36$ cells that bind dengue type 4 virus. J Virol. 1997;71(10):7246-52.

92. Yazi Mendoza $\mathrm{M}$ et al. A putative receptor for dengue virus in mosquito tissues: localization of a 45-kDa glycoprotein. Am J Trop Med Hyg. 2002;67(1):76-84

93. Mercado-Curiel RF et al. The four serotypes of dengue recognize the same putative receptors in Aedes aegypti midgut and Ae. albopictus cells. BMC Microbiol. 2006;6:85.

94. Munoz ML et al. Putative dengue virus receptors from mosquito cells. FEMS Microbiol Lett. 1998;168(2):251-8.

95. Mercado-Curiel RF, Black WCT, Munoz Mde L. A dengue receptor as possible genetic marker of vector competence in Aedes aegypti. BMC Microbiol. 2008;8:118.

96. Cao-Lormeau VM. Dengue viruses binding proteins from Aedes aegypti and Aedes polynesiensis salivary glands. Virol J. 2009;6:35. 